\title{
BmKn-2 Scorpion Venom Peptide for Killing Oral Cancer Cells by Apoptosis
}

\author{
Pirut Tong-ngam ${ }^{1}$, Sittiruk Roytrakul ${ }^{2}$, Hathaitip Sritanaudomchai ${ }^{3 *}$
}

\begin{abstract}
Scorpion venom peptides recently have attracted attention as alternative chemotherapeutic agents that may overcome the limitations of current drugs, providing specific cytotoxicity for cancer cells with an ability to bypass multidrug-resistance mechanisms, additive effects in combination therapy and safety. In the present study, BmKn-2 scorpion venom peptide and its derivatives were chosen for assessment of anticancer activities. BmKn-2 was identified as the most effective against human oral squamous cells carcinoma cell line (HSC-4) by screening assays with an $\mathrm{IC}_{50}$ value of $29 \mu \mathrm{g} / \mathrm{ml}$. The BmKn-2 peptide killed HSC-4 cells through induction of apoptosis, as confirmed by phase contrast microscopy and RT-PCR techniques. Typical morphological features of apoptosis including cell shrinkage and rounding characteristics were observed in treated HSC-4 cells. The results were further confirmed by increased expression of pro-apoptotic genes such as caspase-3, -7 , and -9 but decrease mRNA level of anti-apoptotic BCL-2 in BmKn-2 treated cells, as determined by RT-PCR assay. In summary, the BmKn-2 scorpion venom peptide demonstrates specific membrane binding, growth inhibition and apoptogenic activity against human oral cancer cells.
\end{abstract}

Keywords: BmKn-2 - scorpion venom peptide -chemotherapeutic agents - apoptosis - oral cancer - anticancer

Asian Pac J Cancer Prev, 16 (7), 2807-2811

\section{Introduction}

Recently, it is very interesting in the use of natural toxin to develop safe and more effective therapeutic agents for cancer treatment (Possani et al., 1999; Liu et al., 2014). Scorpion venom peptides have a vast variety of biological activities, immune-modulatory activities and pharmacological functions. The list of scorpion peptides have been expanding to display anti-proliferative and cytotoxic effects on tumor and malignant cells, and new substances are being continually added (Wang and $\mathrm{Ji}$, 2005; Das Gupta et al., 2007; Fu et al., 2012). BmKn-2 is a cationic peptide which isolates from the venom of scorpion Mesobuthus martensii Karsch. The BmKn-2 is a basic, alpha-helical peptide with an amidated C-terminus (Cao et al., 2012) which has strong antimicrobial activity against both Gram-positive and Gram-negative bacteria including Bacillus subtillis, Escherichia coli, Micrococcus luteus, Pseudomonas aeroginosa, and Staphylococcus aureus (Zeng et al., 2004). Moreover, in our previous study (Arpornsuwan et al., 2014), it has been shown that BmKn-2 inhibits the proliferation of several cancer cell types but has lower cytotoxicity to dental pulp stem cells and red blood cells.

Oral cancer is one of the most common causes of cancer-related mortality worldwide (Krishna Rao et al., 2013). The death rate is higher than other common cancer types such as cervical cancer, lymphoma, cancer of the testes, and endocrine system cancers includes thyroid, or skin cancer (Laronde et al., 2008). Oral cancer is particularly dangerous because in its early stages it may not be noticed by the patient, as it can frequently prosper without producing pain or symptoms they might readily recognize, and because it has a high risk of producing second primary tumors (Reis et al., 2011; Krishna Rao et al., 2013). Treatment is with a combination of surgery, radiotherapy and chemotherapy. Chemotherapeutic agents are commonly used as antibiotic or anticancer drugs but they frequently affect normal human cells and cause severe side effects (Gerber, 2008; Weeks et al., 2012). Moreover, cancer cells can develop resistance to conventional chemotherapy agents by cellular changes through multimechanism (Gatti and Zunino, 2005). Development of chemotherapeutic agents that can be used for effective, specific and safe treatment has gained interest.

The effective peptides that exhibit maximum biological activity and minimum cytotoxicity to normal cells should have the optimum of various parameters, such as amino acid sequence, the number of charges, hydrophobicity residues, secondary structure, and position of cationic residues (Khandelia et al., 2008; Pasupuleti et al., 2012). The main aim of this study was to investigate the effect of synthetic BmKn-2 scorpion venom peptide and its derivatives on the viability and the role of the anticancer

${ }^{I}$ Institute of Molecular Biosciences, Nakhon Pathom, ${ }^{2}$ Genome Institute, National Center for Genetic Engineering and Biotechnology, Pathumthani, ${ }^{3}$ Department of Oral Biology, Faculty of Dentistry, Mahidol University, Bangkok, Thailand * For correspondence: hathaitip.sri@mahidol.ac.th 
activity in human oral squamous cells carcinoma HSC-4 cells.

\section{Materials and Methods}

\section{Peptides}

BmKn-2 and its eight derivatives peptides (BmKn-21, BmKn-22, BmKn-23, BmKn-24, BmKn-25, BmKn-26, BmKn-2CC, and cyclic BmKn-2) were synthesized by China peptides Co. LTD, Shanghai, China. Their properties are listed in Table 1. One hundred milligrams of all synthetic peptides were dissolved in $1 \mathrm{ml}$ of dimethyl sulfoxide (DMSO: Sigma, St Louis, MO, USA) and further diluted in oral cancer cells culture medium to obtain desired concentrations.

\section{Maintenance of HSC-4 cells}

The human oral squamous cells carcinoma HSC-4 line was a kind gift from Dr. Rudee Surarit, Faculty of Dentistry, Mahidol University. The cell line was maintained and propagated in Dulbecco's modified Eagle's medium (DMEM; Gibco, Rockville, MD, USA) supplemented with $10 \%$ fetal bovine serum (FBS; Hyclone, Logan, UT, USA) and $1 \%$ penicillin/streptomycin (Gibco). Cells were cultured as adherent mono-layers (i.e., cultured at $\sim 70 \%$ to $80 \%$ confluence) in a humidified atmosphere with $5 \%$ $\mathrm{CO} 2$ at $37^{\circ} \mathrm{C}$. Cells were harvested after being subjected to brief trypsinization.

\section{Measurement of cell viability by MTT}

The modified rapid colorimetric MTT assay was performed to assess the cell viability of different concentrations of synthetic peptides on HSC-4 cells as described previously (Ferrari et al., 1990). Briefly, HSC-4 cells were seeded in 96-well plates at $2 \times 10^{4}$ cells per well in DMEM culture medium for $24 \mathrm{~h}$. Four concentrations $(5,10,50$ and $100 \mu \mathrm{g} / \mathrm{ml})$ of each peptide for overnight treatment were used to determine the $\mathrm{IC}_{50}$ growth inhibitory values of peptide in cancer cells. After incubation, $100 \mu 1$ of MTT [3-(4,5-dimethylthiazol-2yl)-2,5-diphenyltetrazolium bromide, Sigma] solution (1 $\mathrm{mg} / \mathrm{ml}$ in culture medium) was added to each well. The plates were then incubated for $2 \mathrm{~h}$ at $37^{\circ} \mathrm{C}$. Intracellular formazan crystals were dissolved by addition of 100 $\mu \mathrm{l}$ of DMSO to each well. After $30 \mathrm{~min}$ incubation at room temperature, the optical density of the samples was determined at $595 \mathrm{~nm}$. Cells treated with $0.1 \%$ DMSO were used as a solvent control, whereas untreated cells were used as an OD control.

\section{Measurement of apoptotic cell death}

Morphology assay: Apoptosis can be identified morphologically based on the criteria described elsewhere (Kerr et al., 1972). HSC-4 cells were treated with synthetic $\mathrm{BmKn}-2$ peptide at $\mathrm{IC}_{50}$ concentration for $24 \mathrm{~h}$. The effect of peptide on cells morphology was determined by light microscopy.

Reverse transcriptase PCR analysis: The expression of apoptotic genes was analyzed by reverse transcriptionPCR (RT-PCR) technology. Total RNA of cells was isolated using RNeasy Mini Kit according to the manufacturer's instruction (QIAGEN, Valencia, CA, USA). Each $1.0 \mu \mathrm{g}$ of the RNA samples was converted to cDNA with SuperScript II Reverse Transcriptase (Invitrogen, Renfrewshire, UK) in a final volume of 20 $\mu \mathrm{l}$. RT reactions were performed at $50^{\circ} \mathrm{C}$ for $50 \mathrm{~min}$ and $70^{\circ} \mathrm{C}$ for $15 \mathrm{~min}$ in Gene Cycler thermal cycler (Bio-Rad, São Paulo, Brazil). The thermal cycle conditions were carried out for 35 cycles at $95^{\circ} \mathrm{C}$ for $30 \mathrm{~s}, 60^{\circ} \mathrm{C}$ for $30 \mathrm{~s}$, and $72^{\circ} \mathrm{C}$ for $30 \mathrm{~s}$. The cDNA was amplified by PCR with the following primers: Caspase (CASP)-3, forward primer 5'-TGTTTGTGTGCTTCTGAGCC-3', reverse primer 5'-CACGCCATGTCATCATCAAC-3'; CASP-7, forward primer 5'-CCAATAAAGGATTTGACAGCC-3', reverse primer 5'-GCATCTGTGTCATTGATGGG-3'; CASP-9, forward primer 5'-CATTTCATGGTGGAGGTGAAG-3', reverse primer 5'-GGGAACTGCAGGTGGCTG-3'; B-cell CLL/lymphoma (BCL)-2, forward primer 5'-ATGTGTGTGGAGACCGTCAA-3', reverse primer 5'-GCCGTACAGTTCCACAAAGG-3'. The house keeping gene, glyceraldehyde-3-phosphate dehydrogenase (GAPDH) was used as an internal control, forward primer 5'-GTCAGTGGTGGACCTGACCT-3', reverse primer 5'-AGGGGTCTACATGGCAACTG-3'. PCR products were analyzed by $1 \%$ agarose gel and visualized by ethidium bromide staining.

\section{Results}

To explore the anticancer effect of BmKn-2 and its derivatives peptides (BmKn-21, BmKn-22, BmKn-23, BmKn-24, BmKn-25, BmKn-26, BmKn-2CC, and cyclic BmKn-2) on human oral cancer HSC-4 cells, the cytotoxic effect of $0-100 \mu \mathrm{g} / \mathrm{ml}$ peptides was determined using MTT assay. After incubation with BmKn-2, BmKn-21, BmKn-22, BmKn-23, BmKn-24 and BmKn-2CC for $24 \mathrm{~h}$, the proliferation of HSC-4 cells was inhibited in a dose-dependent manner. However, BmKn-25, BmKn-26 and cyclic BmKn-2 peptides showed no significant cell death at the use peptide concentrations on HSC-4 cells, compared to the control (Figure 1). From the experiment, BmKn-2 and BmKn-22 which could only exhibit 50\%

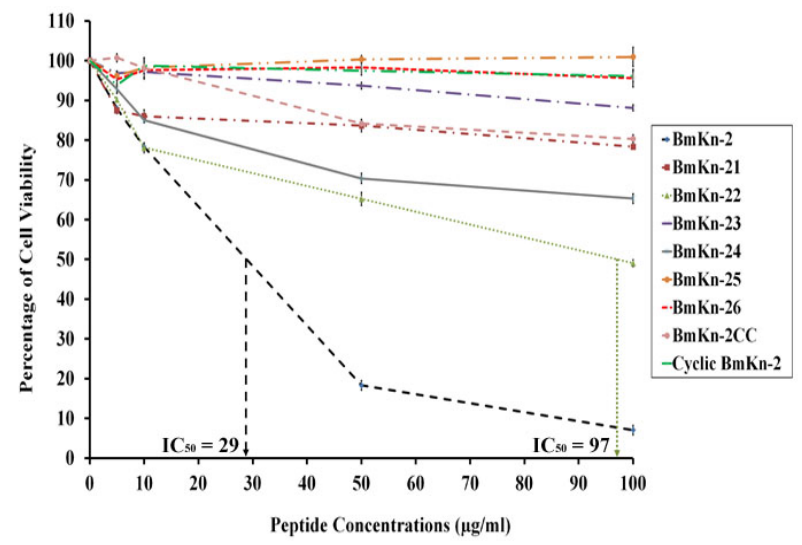

Figure 1. Effect of BmKn-2 Scorpion Venom Peptide and its Derivatives on Growth of Human HSC-4 Cells after $24 \mathrm{~h}$ of Treatment. Cell cytotoxicity was measured using MTT assay. Values are expressed as mean value of cell viability (\% of control) \pm S.D. of three independent experiments. $\mathrm{IC}_{50}$ is the half maximal inhibitory concentration 
Table 1. Physiochemical Properties of BmKn-2 and its Derivatives

\begin{tabular}{llcl}
\hline Peptide name & Amino acid sequence & Net charge & Structure prediction \\
\hline BmKn-2 & FIGAIARLLSKIF & +2 & CHHHHHHHHHHCC \\
BmKn-21 & FIGAIARLLSKI & +2 & CHHHHHHHHCC \\
BmKn-22 & FIGAIARLLSK & +2 & CCHHHHHHCC \\
BmKn-23 & FIGAIARLLS & +1 & CHHHHHHCC \\
BmKn-24 & FIGAIARLL & +1 & CHHHHHHCC \\
BmKn-25 & FIGAIARL & +1 & CCCCCCC \\
BmKn-26 & FIGAIAR & +1 & CCCCCC \\
BmKn-2CC & CFIGAIARLLSKIFC & +2 & CHHHHHHHHHHHHC \\
Cyclic BmKn-2 & FIGAIARLLSKIF (N to C cyclization) & +2 & ND \\
\hline
\end{tabular}

$\mathrm{C}$, random coil; $\mathrm{H}$, helix; ND, not determined

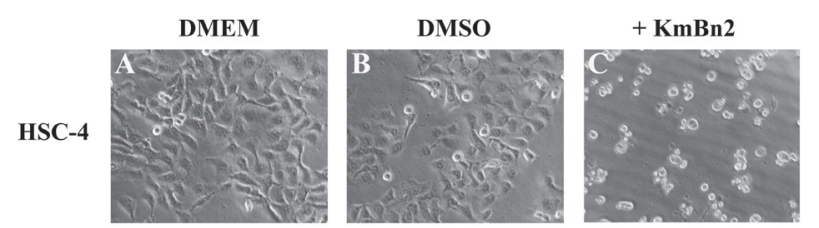

Figure 2. Phase Contrast Microscopy Images of Control HSC-4 Cells (DMEM), 0.1\% DMSO-treated HSC-4 and $29 \mu \mathrm{g} / \mathrm{ml} \mathrm{BmKn-2-treated} \mathrm{HSC-4.}$ Magnification (100x)

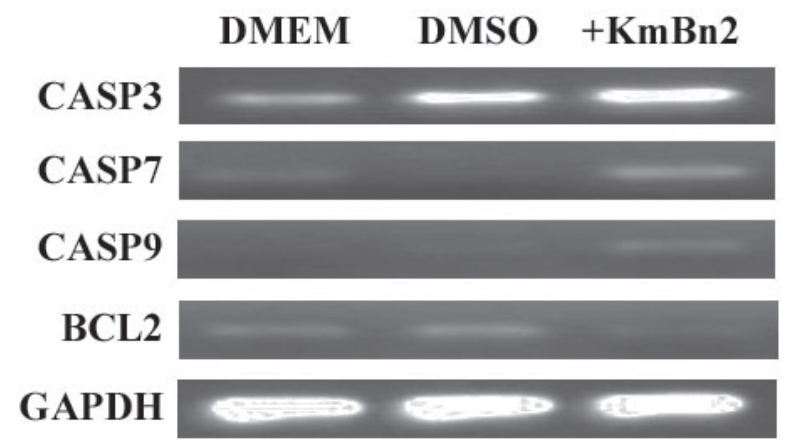

Figure 3. Expression of Apoptosis Related Genes after 24h Exposure of BmKn-2 Peptide. HSC-4 cells were treated with synthetic BmKn-2 peptide at $\mathrm{IC}_{50}$ concentration and analyzed by RT-PCR. PCR products were analyzed by $1 \%$ agarose gel and visualized by ethidium bromide staining

inhibition of cell growth $\left(\mathrm{IC}_{50}\right)$ that were 29 and $97 \mu \mathrm{g} /$ $\mathrm{ml}$, respectively (Figure 1). These data suggest that HSC4 cells were more susceptible to BmKn-2 than other selective peptides.

BmKn-2 was further chosen since this was the peptide which had robust growth inhibition effect on human oral cancer cells HSC-4. BmKn-2 peptide has been designed to decrease the net positive charge by sequential shortening from the C-termini (Table 1). The secondary structures have been predicted by PHD method (Rost and Sander, 1993) on the website (http://npsa-pbil.ibcp.fr/cgi-bin/npsa/ automat.pl?page=/NPSA/npsa.phd.html), suggesting that the percentage of alpha-helix from $\mathrm{BmKn} 2$ has higher than its C-terminally truncated derivatives $(\mathrm{BmKn}-21$, BmKn-22, BmKn-23, BmKn-24) but lower than BmKn2CC. However, BmKn-25 and BmKn-26 have contained no alpha-helix as describe previously (Arpornsuwan et al., 2014) (Table 1). To determine whether the BmKn-2 induced loss of HSC-4 cells viability was associated with the induction of apoptosis, morphology assay and mRNA levels of apoptotic-related genes were used. Microscopic observation showed that human oral squamous cells carcinoma HSC-4 cells grown as monolayer overnight in cell culture medium DMEM or 0.1 \% DMSO (Control; Figure 2A, 2B). On the other hand, HSC-4 cells lost their ability to adhere to surfaces of tissue culture plates after $\mathrm{BmKn}-2$ peptide treatment at $\mathrm{IC}_{50}$ concentration (Figure 2C). In addition, the peptide induced HSC-4 cells morphological changes such as cell shrinkage and rounded shape formation (Figure 2C). We further investigated the molecular mechanism of BmKn-2 induced apoptosis in HSC-4 cell, the expression levels of several apoptosisrelated genes were examined. Figure 3 summarized the gene expression changed of Caspase-3, -7, -9 (CASP-3, $-7,-9$ ) and BCL-2. The CASP-3 transcripts of HSC-4 cells were gradually increased by culturing in DMEM (culture medium), $0.1 \%$ DMSO and $\mathrm{KmBn}-2$ peptide, respectively (Figure 3). Similarly, the expression levels of CASP-9 in BmKn-2 treated HSC-4 cells increased when compared to control (Figure 3). Moreover, the expression levels of CASP-7 gene in oral cancer cells treated with control solvent DMSO for $24 \mathrm{~h}$ slightly decreased when compared to untreated control cells (DMEM). While HSC-4 cells treated with BmKn-2 markedly increased the CASP-7 mRNA level (Figure 3). Conversely, after incubation with DMSO, the band of anti-apoptotic BCL-2 mRNA expression was more intense band than control DMEM in HSC-4 cells. However, BCL-2 gene band of treated cells with $\mathrm{BmKn}-2$ peptide was showing very faint when compared to control (Figure 3).

Together, these data suggest that BmKn-2 peptide were effective in killing the human oral squamous cells carcinoma HSC-4 by an apoptotic process. BmKn-2 peptide may provide an effective therapy in pre-clinical oral cancer.

\section{Discussion}

Unfavorable side effects and resistance to many anticancer agents that have been developed are serious problems (Possani et al., 1999). Here, we first report the effects of BmKn-2 and its eight derivatives peptides on oral cancer and its mechanisms of action. From selected nine peptides, $\mathrm{BmKn}$-2 was the most effective inhibition of human oral squamous cells carcinoma HSC-4 proliferation whereas the peptide has lower cytotoxicity to dental pulp stem cells and red blood cells (Arpornsuwan et al., 2014). In contrast, it shows growth inhibition of normal smooth muscle at the low concentration (Arpornsuwan et al., 2014), suggesting the peptide was very high cell 
selectivity. The susceptibility and selectivity of BmKn-2 against normal and cancer cells could be related to the presence of specific molecules on the cell surface. One of the major differences between cancer and non-cancer cell external surfaces is the exposure of the negatively charged molecules. Generally, tumor cell membranes contain 3-7 times more phosphatidylserine than normal cells (Chiu et al., 1979; Dobrzynska et al., 2005). Secondary structure prediction showed that BmKn-2 contains one alpha-helix domain, and two flexible random coiled regions at both terminus (Arpornsuwan et al., 2014). BmKn-2 peptide has a distinct hydrophobic and hydrophilic region, making the molecules markedly amphipathic (Zeng et al., 2004), which may allow BmKn-2 to bind and break the membrane of oral cancer HSC-4 cells. Here, the cytotoxic effect of BmKn-2 on neoplastic cells is commonly believed to be a function of the cationic nature and secondary structure of the peptide. It is possible that BmKn-2 has a unique cell penetrating property across the membrane and killing cancer cells by acting on intracellular targets.

Apoptosis or programmed cell death is beneficial in cancer therapy (Chinkwo, 2005; Li et al., 2014; Sun et al., 2014; Wang et al., 2014). Cationic antimicrobial peptides induce $\mathrm{Ca} 2+$ influx in tumor cells followed by DNA fragmentation characteristic of apoptosis or binding to receptor followed by interacting with multiple SH3containing cytoplasmic proteins (Chan and Gallo, 1998; Risso et al., 1998; Tanaka et al., 2001). The further study was conducted to evaluate apoptogenic activity of BmKn2 on human oral cancer cell line HSC-4. Apoptosis can be initially distinguished by characteristic changes in cell morphology. These include cell shrinkage, chromatin condensation, and nuclear fragmentation cleavage (Kerr et al., 1972). The induction of apoptosis by BmKn-2 was evidenced from the morphological alteration such as a diminished size and became more circular in shape in comparison with untreated HSC-4 cells. There are many mechanisms through which apoptosis can be induced in cells. Two main evolutionarily conserved gene and protein families are involved in apoptosis, namely the cysteinyl aspartate-specific protease or caspases, which mediate the execution phase of apoptosis (Fuentes-Prior and Salvesen, 2004), and the BCL-2 family, which control mitochondrial integrity (Youle and Strasser, 2008). Hence, the level of apoptosis in a particular HSC-4 cell population was estimated from the mRNA levels of several apoptosisrelated genes (Caspase-3, -7, -9 and BCL-2). Caspases (CASP) have a central role in the apoptotic process in that they trigger a cascade of apoptotic pathways (Shah et al., 2003). In mammalian cells, the release of cytochrome c from mitochondria leads to the activation of procaspase-9. Active CASP-9 cleaves and activates the downstream executioner CASP-3, -6 , and -7 , which are crucial for the execution of apoptotic cell death (Hengartner, 2000). The data presented in this study indicate that $\mathrm{BmKn}-2$ peptide possessed apoptogenic effect on HSC-4 oral cancer cell line by increasing the expression levels of caspase-3, -7 and -9 when compared to control. On the other hand, anti-apoptotic BCL-2 mRNA level was depressed after BmKn-2 peptide treatment. DNA damage and cytotoxicity insults are acted through the mitochondria, are regulated by the BCL- 2 family (Youle and Strasser, 2008). The anti-apoptotic Bcl-2 family members maintain mitochondrial integrity by preventing the pro-apoptotic multi-domain Bcl-2 family member Bax and Bak from causing mitochondrial damage (Riedl and Salvesen, 2007). Taken together, the data presented in this study suggest that BmKn-2 induced apoptosis is mediated by the death receptor and mitochondrial apoptotic pathways.

In conclusion, to our knowledge, the results presented in this study show for the first time that the synthetic cationic BmKn-2 scorpion venom peptide exhibits selective cytotoxic activity against human oral squamous cells carcinoma HSC-4 cell. Moreover, $\mathrm{BmKn}-2$ peptide induces apoptosis in HSC-4 by modulating expression levels of caspase-3, -7, and -9 and BCL-2. Therefore, the synthetic BmKn-2 peptide provides the underlying mechanism for the anti-oral cancer activity, making it a promising chemotherapeutic agent for oral cancer treatment; this is likely to be confirmed by further investigation.

\section{Acknowledgements}

The authors would like to acknowledge the Faculty of Dentistry and Institute of Molecular Biosciences, Mahidol University and National Center for Genetic Engineering and Biotechnology, Thailand for providing expertise and services that contributed to this project. Funding was provided by Mahidol University Faculty of Dentistry Grant (2014).

\section{References}

Arpornsuwan T, Buasakul B, Jaresitthikunchai J, et al (2014). Potent and rapid antigonococcal activity of the venom peptide BmKn2 and its derivatives against different Maldi biotype of multidrug-resistant Neisseria gonorrhoeae. Peptides, 53, 315-20.

Arpornsuwan T, Sriwai W, Jaresitthikunchai J, et al (2014). Anticancer activities of antimicrobial BmKn2 peptides against oral and colon cancer cells. Int J Pept Res Ther, 20, 501-9.

Cao L, Dai C, Li Z, et al (2012). Antibacterial activity and mechanism of a scorpion venom peptide derivative in vitro and in vivo. PLoS One, 7, 40135.

Chan YR, Gallo RL (1998). PR-39, a syndecan-inducing antimicrobial peptide, binds and affects p130(Cas). J Biol Chem, 273, 28978-85.

Chinkwo KA (2005). Sutherlandia frutescens extracts can induce apoptosis in cultured carcinoma cells. J Ethnopharmacol, 98, 163-70.

Chiu D, Lubin B, Shohet SB (1979). Erythrocyte membrane lipid reorganization during the sickling process. Br J Haematol, 41, 223-34.

Das Gupta S, Debnath A, Saha A, et al (2007). Indian black scorpion (Heterometrus bengalensis Koch) venom induced antiproliferative and apoptogenic activity against human leukemic cell lines U937 and K562. Leuk Res, 31, 817-25.

Dobrzynska I, Szachowicz-Petelska B, Sulkowski S, et al (2005). Changes in electric charge and phospholipids composition in human colorectal cancer cells. Mol Cell Biochem, 276, 113-9.

FerrariM, Fornasiero MC, Isetta AM, et al (1990). MTT colorimetric assay for testing macrophage cytotoxic activity in vitro. J Immunol Methods, 131, 165-72. 
Fu Y, Zheng S, Huang R, et al (2012). A potential strategy for high-grade gliomas: combination treatment with lithium chloride and BmK CT. Biotechnol Lett, 34, 9-17.

Fuentes-Prior P, Salvesen GS (2004). The protein structures that shape caspase activity, specificity, activation and inhibition. Biochem J, 384, 201-32.

Gatti L, Zunino F (2005). Overview of tumor cell chemoresistance mechanisms. Methods Mol Med, 111, 127-48.

Gerber DE (2008). Targeted therapies: new generation of cancer treatments. Am Fam Physician, 77, 311-9.

Hengartner MO (2000). The biochemistry of apoptosis. Nature, 407, 770-6.

Kerr JF, Wyllie AH, Currie AR (1972). Apoptosis: a basic biological phenomenon with wide-ranging implications in tissue kinetics. Br JCancer, 26, 239-57.

Khandelia H, Ipsen JH, Mouritsen OG (2008). The impact of peptides on lipid membranes. Biochim Biophys Acta, 1778, 1528-36.

Krishna Rao SV, Mejia G, Roberts-Thomson K, et al (2013). Epidemiology of oral cancer in Asia in the past decade-an update (2000-2012). Asian Pac J Cancer Prev, 14, 5567-77.

Laronde DM, Hislop TG, Elwood JM, et al (2008). Oral cancer: just the facts. J Can Dent Assoc, 74, 269-72.

Li J, Bian WH, Wan J, et al (2014). Curdione inhibits proliferation of MCF-7 cells by inducing apoptosis. Asian Pac J Cancer Prev, 22, 9997-10001.

Liu CC, Yang H, Zhang LL, et al (2014). Biotoxins for cancer therapy. Asian Pac J Cancer Prev, 12, 4753-8.

Pasupuleti M, Schmidtchen A, Malmsten M (2012). Antimicrobial peptides: key components of the innate immune system. Crit Rev Biotechnol, 32, 143-71.

Possani LD, Becerril B, Delepierre M (1999). Scorpion toxins specific for Na+-channels. Eur JBiochem, 264, 287-300.

Reis PP, Waldron L, Perez-Ordonez B (2011). A gene signature in histologically normal surgical margins is predictive of oral carcinoma recurrence. BMC Cancer, 11, 437.

Riedl SJ, Salvesen GS (2007). The apoptosome: signalling platform of cell death. Nat Rev Mol Cell Biol, 8, 405-13.

Risso A, Zanetti M, Gennaro R(1998). Cytotoxicity and apoptosis mediated by two peptides of innate immunity. Cell Immunol, 189, 107-15.

Rost B, Sander C (1993). Prediction of protein secondary structure at better than $70 \%$ accuracy. $J$ Mol Biol, 232, 584-99.

Shah S, Gapor A, Sylvester PW (2003). Role of caspase-8 activation in mediating vitamin E-induced apoptosis in murine mammary cancer cells. Nutr Cancer, 45, 236-46.

Sun J, Zhang C, Bao YL, et al (2014). Parthenolide-induced apoptosis, autophagy and suppression of proliferation in HepG2 cell. Asian Pac J Cancer Prev, 12, 4897-902.

Tanaka K, Fujimoto Y, Suzuki M (2001). PI3-kinase p85alpha is a target molecule of proline-rich antimicrobial peptide to suppress proliferation of ras-transformed cells. Jpn J Cancer Res, 92, 959-67.

Wang WX, Ji YH (2005). Scorpion venom induces glioma cell apoptosis in vivo and inhibits glioma tumor growth in vitro. J Neurooncol, 73, 1-7.

Wang G, Jiang MY, Meng Y, et al (2014). Cellular mechanisms of a new pyrazinone compound that induces apoptosis in SKOV-3 cells. Asian Pac J Cancer Prev, 2, 797-802.

Weeks JC, Catalano PJ, Cronin A, et al (2012). Patients' expectiations about effects of chemotherpy for advanced cancer. $N$ Engl J Med, 367, 1616-25.

Youle RJ, Strasser A (2008). The BCL-2 protein family: opposing activities that mediate cell death. Nat Rev Mol Cell Biol, 9, 47-59.

Zeng XC, Wang SX, Zhu Y (2004). Identification and functional characterization of novel scorpion venom peptides with no disulfide bridge from Buthus martensii Karsch. Peptides, 25, 143-50. 